\title{
Lung Damage among Individuals with Biomass Smoke Exposure Characterized by CT Features and Changes in Pulmonary Function
}

\section{Jinglong Chen}

Guangzhou First People's Hospital https://orcid.org/0000-0001-6042-0136

Changbin Jiang

Guangzhou Institute of Respiratory Disease

\section{Youlan Zheng}

Guangzhou Institute of Respiratory Disease

\section{Dongxing Zhao}

Guangzhou Institute of Respiratory Disease

\section{Fan Wu}

Guangzhou Institute of Respiratory Disease

\section{Zhuxiang Zhao}

Guangzhou First People's Hospital

\section{Jun Zhao}

Guangzhou First People's Hospital

\section{Qin Li}

Guangzhou First People's Hospital

\section{Bing Li}

Guangzhou Medical University

\section{Gongyong Peng}

Guangzhou Institute of Respiratory Disease

\section{Yumin Zhou}

Guangzhou Institute of Respiratory Disease

Pixin Ran ( $\nabla$ pxran@gzhmu.edu.cn )

Guangzhou Institute of Respiratory Disease https://orcid.org/0000-0001-6651-634X

\section{Research}

Keywords: biomass smoke, chronic obstructive pulmonary disease, computed tomography, pulmonary function.

Posted Date: June 16th, 2021 
DOl: https://doi.org/10.21203/rs.3.rs-586331/v1

License: (c) (1) This work is licensed under a Creative Commons Attribution 4.0 International License. Read Full License 


\section{Abstract}

\section{Background}

Biomass smoke exposure (BSE) is an important etiological factor in chronic obstructive pulmonary disease (COPD). However, few studies have focused on the effects of BSE in the respiratory muscles or lungs.

\section{Methods}

Using a cohort, we selected 98 participants in underdeveloped rural areas: 16 healthy individuals with BSE ("BSE normal"), 19 patients with BSE and COPD ("BSE+COPD"), 13 healthy individuals with cigarette smoke exposure ("CSE normal"), 25 patients with cigarette smoke exposure and COPD ("CSE+COPD"), and 25 healthy controls. Patients with GOLD stage I and II COPD were included. Baseline data (demographic data, BSE or CSE, lung function, and CT findings) and follow-up lung function data were collected. CT parameters of emphysema, pulmonary small vessels, airway remodeling, pectoralis muscles, and erector spinae muscle were measured.

\section{Results}

Individuals with BSE were mainly women (32/35, 91.43\%). Compared with the CSE+COPD group, the BSE+COPD group demonstrated slow lung function decline, increased lower lung emphysema, and narrower airway lumen and airway wall thickening in moderate and small airways (all $P<.05$ ). Compared with healthy controls, the CSE normal and BSE normal groups exhibited significant reduction of pulmonary small vessel area and obvious airway remodeling in small airways $(P<.05)$. Compared with the BSE normal group, the BSE+COPD group showed significantly more severe emphysema and airway remodeling, as well as reduced left pectoralis major muscle area (all $P<.05$ ).

\section{Conclusions}

Healthy individuals with BSE had reduced pulmonary small vessel area and obvious airway remodeling; patients with BSE and COPD showed more severe emphysema, airway remodeling, and pectoralis major muscle change. More investigations are needed regarding interventions for BSE.

Trial registration: ChiCTR, ChiCTR-00-14004264 . Registered 12 February 2014, http://www.chictr.org.cn/showproj.aspx?proj=5304

\section{Background}

Chronic obstructive pulmonary disease (COPD) is associated with exposure to large amounts of toxic particles or gases. Biomass smoke exposure (BSE) and cigarette smoke exposure (CSE) are important risk factors for COPD [1, 2]. In recent decades, considerable progress has been made in the treatment of COPD, but its incidence and mortality remain high [1, 2], which suggests that COPD-related environmental 
and occupational factors require further improvement [3]. Among these factors, the absence of interventions for BSE is particularly obvious [4]. Nearly 3 billion people worldwide use biomass as energy for heating and cooking, which results in substantial BSE [1]. Individuals affected by BSE mainly comprise women who use biomass for cooking in underdeveloped rural areas; these individuals tend to have relatively low socioeconomic statuses [5-8].

To the best of our knowledge, there has been insufficient attention to BSE. A few studies have performed analyses of clinical symptoms and the CT findings of total proportions of emphysema and small airway disease caused by BSE [9-12]. There is a lack of comprehensive studies regarding pulmonary lesions caused by BSE, how BSE affects the pulmonary small vessels, the respiratory muscles, the development of emphysema and airway remodeling. The dangers of BSE have received less publicity in social media, compared with the dangers of CSE [12-15]. For example, among users of TikTok (a popular social media service in China), there are currently 198 accounts that promote smoking cessation-related content; by contrast, only one professional account addresses the risks of biomass smoke pollution. Similarly, the efficacies of clean energy replacement plan are sometimes disappointing; these plans are influenced by factors such as economics, education, and women's social status in the family $[\underline{5}, \underline{6}, \underline{8}, \underline{16}$. These data suggest that the task of reducing BSE cannot be accomplished by individuals or families, requires societal mobilization for effective outcomes [17].

Computed tomography (CT)-derived evaluation of the basic structural changes of COPD (e.g., airway remodeling, emphysema, pulmonary vascular changes, and respiratory muscles) can reflect the severity of lung lesions, thus facilitating objective assessment and prediction of disease progression [18-22]. Our study focused on populations with BSE in economically underdeveloped rural areas to comprehensively investigate CT findings in patients with and without COPD and exposures to cigarette smoke or biomass fuels. This study aimed to clarify the features of BSE-related lung injury compared to CSE induced injury that results in COPD vs those without COPD.

\section{Methods}

\section{Study design}

The Ethics Committee of Guangzhou Institute of Respiratory Health approved the study protocol. Participants were selected from among the rural population in an underdeveloped mountainous area of northern Guangdong, China, using an observatory research subgroup data from COPD community screening database (from 2014 to 2015) [10 23, 24]. No intervention was performed on the whole participants and no medication was prescribed to patients with COPD. BSE exposure population and CSE population were followed for a year. Healthy control individuals were not followed. Written informed consent was obtained from all participants. Detailed information regarding recruitment, clinical management, and follow-up was described in previous studies $[10, \underline{23}, \underline{24}]$.

\section{Population}


This study included individuals with BSE or CSE (including otherwise healthy individuals and patients with COPD) and healthy controls, who were over $>40$ years of age, had complete clinical data, pulmonary function assessments, and lung HRCT data. The study excluded patients who had lung cancer, asthma, interstitial lung disease, pulmonary infarction, pneumonia, or pleural effusion,based on medical history or CT scan findings. The healthy control group included healthy individuals with normal lung function, without a history of CSE or BSE, without a history of pulmonary disease or respiratory symptoms, and without visible emphysema or low attenuation areas less than a threshold of -950 Hounsfield units (LAA$950 \%)<5 \%$ in CT scans.

\section{Patient Groups}

According to their history of CSE or BSE and whether they had been diagnosed with COPD, 98 eligible participants were allocated into five groups: BSE+COPD ( $n=19)$, BSE normal $(n=16), \operatorname{CSE}+\operatorname{COPD}(n=25)$, CSE normal ( $n=13)$, and healthy control $(n=25)$ (Figure 1$)$. The BSE+COPD and CSE+COPD groups only included patients with Global Initiative for Chronic Obstructive Lung Disease (GOLD) stages I and II (postbronchodilator forced expiratory volume in 1 second $\left[\mathrm{FEV}_{1}\right] /$ forced vital capacity $[\mathrm{FVC}]<0.7$ and $\mathrm{FEV}_{1} \geq$ $50 \%$ predicted).

\section{Data collected}

Baseline records of participants were collected, including demographic information, lung function, history of BSE and/or CSE, and high-resolution CT findings; follow-up lung function data were also collected. The BSE and CSE indexes were calculated as previously described [10]. The BSE index is defined as the cumulative exposure of biomass, which is calculated by multiplying the average number of hours per day in the kitchen with the number of years of cooking with biomass.Cigarette smoking index was expressed as pack-years.

Annual reductions in lung function ( $\mathrm{FEV}_{1}$ and $\mathrm{FVC}$ ) were calculated. High-resolution $\mathrm{CT}$ findings were assessed to determine the progression of emphysema and airway remodeling, as well as damage to pulmonary small vessels, pectoralis major muscle, pectoralis minor muscle, and erector spinae muscle.

\section{Imaging methods}

\section{Quantitative CT imaging analysis}

High-resolution CT was performed at suspended full inspiration using a multidetector row CT scanner (Aquilion 16, Toshiba, Tokyo, Japan) [10]. CT scan findings were analyzed with 3D slicer software, version 4.8.1 (https://www.slicer.org, Brigham and Women's Hospital).

\section{CT measurement of emphysema}

Emphysema was detected using the chest imaging platform/parenchyma module of 3D slicer software, using a Hounsfield unit threshold of -950 (i.e., \%LAA-950). The (upper third)/(lower third) ratio of LAA- 
950 was used to assess the distribution of emphysema[18, 25].

\section{CT measurement of bronchial inner diameter and bronchial wall thickness}

Using the Airway Inspector module of 3D slicer software [19], the third, fourth, fifth, sixth generation of airway bronchi of the right upper lobe apical bronchus (i.e., RB1) and right lower lobe posterior bronchus (i.e., RB10) were identified. Then, the inner diameter, bronchial wall thickness, and bronchial wall area\% were detected.

\section{CT measurement of cross-sectional area (CSEA)\% of pulmonary small vessels}

In accordance with the method described by Matsuoka et al $[19, \underline{26}]$, pulmonary small vessels were defined as "circular" blood vessels that were perpendicular to the cross-sectional plane and exhibited a cross-sectional area of $<5 \mathrm{~mm}^{2}$. The upper, middle, and lower slices were captured $1 \mathrm{~cm}$ above the top of the aortic arch, $1 \mathrm{~cm}$ below the carina, and $1 \mathrm{~cm}$ below the right lower pulmonary vein, respectively. CSEA $_{<5 \%}$ was calculated as the percentage of small vessel CSEA to lung CSEA in each slice. The total CSEA $_{<5 \%}$ was calculated as the percentage of total small vessel CSEA to total lung CSEA of three slices.

CSEA\% detection was performed as shown in Figure 2. First, the segmentation and modeling of the lungs were conducted using the Parenchyma Analysis and edit modules in the 3D slicer software. The segmented lung areas were filled with -200 to achieve a uniformly gray color. Second, chest vessels were segmented using the VTMK module in 3D slicer software. Pulmonary vessels were segmented by clipping chest vessels with the lung model. Third, in the above three slices, images of pulmonary vessels and gray lungs were saved separately. Image-Pro Plus 6.0 software (Media Cybernetics, Inc.) was used to measure the areas of gray lungs and small pulmonary vessels. Fourth, pulmonary vessels with "Rounders" of 0.91 (i.e.,circular blood vessels) and "Areas" of $\leq 5 \mathrm{~mm}^{2}$ were segmented as pulmonary small vessels $\left(\mathrm{CSEA}_{<5}\right)$. Fifth, CSEAs of gray lungs and small vessels were measured using the count/size module in Image-Pro Plus software for each slice. Finally, $\mathrm{CSEA}_{<5 \%}$ in each slice and total $\operatorname{CSEA}_{<5 \%}$ were calculated using the formula described above.

\section{CT measurement of pectoralis and erector spinae muscles}

The area and density of muscles were measured using the body composition module of 3D slicer software, as shown in Figure 3. The pectoralis major and minor muscles were measured at the first slice above the aorta, while the erector spinae muscle was measured at the lower slice of the T12 thoracic vertebra [21, 27].

\section{Statistical analysis}

Data are presented as mean \pm standard deviation, median (quartile), or numbers. Comparisons of continuous variables among groups or between multiple groups were evaluated by one-way analysis of variance with a post hoc test (least significant difference method) , Kruskal-Wallis test or Mann-Whitney 
U test . Comparisons of categorical variables among groups were performed using Fisher's exact test. Pearson's correlation coefficients were used to assess associations between lung function decline and CT index. Statistical analyses were conducted using IBM SPSS Statistics, version 23.0 (IBM Corp., Armonk, NY, USA). Two-tailed $P$-values $<.05$ were considered statistically significant.

\section{Results}

\section{Baseline characteristics}

Supplementary Table 1 describes the baseline characteristics of participants in each group. This study focused on economically underdeveloped mountainous rural areas, where individuals with BSE were mainly women who cook with biofuel, while smokers were mainly men. The BIOFUEL-index for BSE COPD and the smoking index for CSE COPD had no significantly difference with BSE normal group and CSE normal groups (P>.05). There were no significant differences in baseline lung function $\left(\mathrm{FEV}_{1} \%\right.$ and $\mathrm{FEV}_{1} / \mathrm{FVC}$ ) and GOLD stage between the BSE+COPD and CSE+COPD groups (both $P>05$ ).

Our previous study demonstrated that BSE COPD patients presented similar respiratory symptom (cough, dyspnoea and wheezing) and similar overall COPD assessment scores (CAT, mMRC and combined COPD assessment) to CSE COPD patients and weaker activity tolerance (six-minute walk distance) [10].This study discovered that lung function decline $\left(\mathrm{FEV}_{1}\right.$ and $\left.\mathrm{FEV}_{1} / \mathrm{FVC}\right)$ was slower in the BSE+COPD group than in the CSE+COPD group (both $P<.05$ ), suggesting that patients with BSE and COPD may exhibit slow progression of disease and experience greater benefit from early active intervention.

\section{CT features of individuals with BSE}

\section{Patients with BSE and COPD exhibited prominent emphysema in the lower lung}

Like patients in the CSE+COPD group, those in the BSE+COPD group had substantial changes in emphysema, and there was no significant difference in the total percentage of emphysema between the two groups (PS.05). However, lower lung emphysema was more obvious in the BSE+COPD group than in the CSE+COPD group $(P<.05)$. See Supplementary Table 2.

\section{Pulmonary small vessel areas were significantly reduced in otherwise healthy individuals with BSE}

Compared with the healthy control group, both the BSE normal and CSE normal groups showed reduced pulmonary small vessel areas $(P<.05)$; this was consistent among the upper, middle, and lower parts of the lung $(P<.05)$. Compared with the BSE normal and CSE normal groups, the pulmonary small vessel areas in $\mathrm{BSE}+\mathrm{COPD}$ and $\mathrm{CSE}+\mathrm{COPD}$ groups were further reduced, but these differences were not statistically significant difference (all $P$ S.05). Finally, there was no significant difference in the reduction of pulmonary small blood vessel areas between the BSE+COPD and CSE+COPD groups (PS.05). See Supplementary Table 2. 


\section{More serious airway remodeling in medium and small airways (grades III-VI bronchi) was observed in the BSE+COPD group}

Small airway remodeling was present in otherwise healthy individuals with BSE, while patients with BSE and COPD exhibited serious medium and small airway remodeling.Compared with the CSE normal group, small airway remodeling (i.e., narrow airway and thickened airway wall) was obvious in grades IV-VI bronchi in the BSE normal group (all $P<.05$ ). Compared with the CSE+COPD group, more serious airway remodeling in medium and small airways (grades III-VI bronchi) was observed in the BSE+COPD group (all $P<$.05). See Supplementary Table 2.

\section{Left pectoralis major muscle area significantly decreased in patients with BSE and COPD}

The pectoralis major muscle area and density were considerably greater in the BSE normal group than in the healthy control group (all $P<.05$ ); similar findings were present in the CSE normal group (all $P<.05$ ). Compared with the BSE normal group, the left pectoralis major muscle area significantly decreased in the BSE+COPD group $(P<0.05)$. Compared with the CSE normal group, the CSE+COPD group tended to show reduced areas of pectoralis and erector spinae muscles, but these differences were not statistically significant (all P>.05).See Supplementary Table 2.

\section{CT presentation and lung function changes}

Lung function decline was relatively slow in the BSE+COPD group; there were no correlations between CT indexes and annual reduction of FEV 1 (all P P.05;Supplementary Table 3). However, the FEV 1 reduction was relatively rapid in the CSE+COPD group; this reduction was negatively correlated with the right pectoralis minor muscle area $(r=-0.68, P<.01$; Supplementary Table 3$)$.

\section{Discussion}

CSE is the most common cause of COPD. The harmfulness of smoking is well-known among members of the public, especially because of social media campaigns and the development of smoking cessation clinics $[14,15, \underline{28}, \underline{29}$. In contrast, the risks of BSE are not well-known among the public and healthrelated investments regarding BSE are insufficient.

This real-world study was conducted in an underdeveloped mountainous area. The composition of the study population is representative of the local area.The study specifically focused on describing the lung damage caused by BSE, with CSE as a reference exposure.

The limited evidence regarding lung damage caused by BSE has mainly emphasized emphysema and air trapping [10-12], which does not provide sufficient insights to guide BSE intervention. In this study, we used 3D slicer software to make an overall assessment of lung damage,including airway remodeling in medium and small airways, emphysema, pulmonary small vessels, and respiratory muscles. Qualitative and quantitative determinations of lung damage caused by BSE were performed following three- 
dimensional and two-dimensional imaging of lung lesions. The resulting representative lung lesion images from individuals with BSE will presumably be useful for further scientific research and public health-related interventions.

Different from previous reports [10,12], we discovered that airway damage was more serious in the BSE+COPD group than in the CSE+COPD group, such that it involved substantial remodeling of small and medium airways. Furthermore, pectoralis major muscle area decreased in the BSE+COPD group, compared with the BSE normal group; a corresponding difference was not observed between CSE+COPD and CSE normal groups. The impacts of BSE on respiratory muscles, compared with CSE, require further investigation.

An interesting finding was that both the BSE+COPD and BSE normal groups had significant reductions in pulmonary small vessel area. To the best of our knowledge, this is the first study to show changes in pulmonary small vessel area among individuals with BSE. The reduction of pulmonary small vessel area is associated with acute exacerbation of COPD [30]and serves as an independent risk factor for mortality[31].Additionally, BSE is associated with elevated risks of hypertension, coronary heart disease, and stroke [32]. Therefore, in addition to pulmonary small vessels, BSE might also be involved in other cardiovascular events.

The present study showed that lung function decline was slightly slower in the BSE+COPD group, which was consistent with the results reported by Salvi et al [33]. Since efforts to improve cooking fuels and kitchen ventilation will reduce indoor $\mathrm{PM}_{2.5}$ concentrations, delay lung function decline, and lower the risk of respiratory disease[24, 34], early intervention for BSE patients may benefit more.

Phenotyping studies regarding COPD have found that specific lung CT features are closely related to the progression of COPD. Specific types of emphysema, airway remodeling, and changes in pulmonary small vessel and chest muscle areas, all of which can be reversed by active intervention, are associated with the progression of COPD $[18, \underline{19}, \underline{21}, \underline{22}, \underline{30}, \underline{31}, \underline{35}, \underline{36}]$. Therefore, we examined correlations between these pathological changes and the annual reduction of $\mathrm{FEV}_{1}$, with the aim of identifying a target to delay lung function decline in patients with BSE and COPD. However, we did not find an association between any CT index and reduction of $\mathrm{FEV}_{1}$ in the BSE+COPD group. This may have been influenced by the small number of participants in the BSE+COPD group, therefore producing no statistically significant findings.

This study had some limitations. First, the number of participants was relatively small, and there were no follow-up lung CT data for any participants. This is just a reflection of the insufficient attention to BSE and limited relevant research funding. Second, this study did not specifically recruit women in the CSE+COPD group or men in the BSE+COPD group; therefore, future studies should focus on sex differences between BSE+COPD and CSE+COPD groups.

\section{Conclusions}


In summary, this study focused on individuals with BSE living in an underdeveloped rural area. It showed that otherwise healthy individuals exposed to BSE had reduced pulmonary small vessel area and obvious airway remodeling, while patients with BSE and COPD had severe emphysema, airway remodeling, and muscle change, as well as relatively slow pulmonary function decline. More investigations are thus needed regarding interventions for BSE.

\section{Abbreviations}

BSE Biomass smoke exposure

COPDChronic obstructive pulmonary disease

CSE Cigarette smoke exposure

CSEA Cross-sectional area

CT Computed tomography

$\mathrm{FEV}_{1}$ Forced expiratory volume in 1 second

FVC Forced vital capacity

GOLD Global Initiative for Chronic Obstructive Lung Disease

\section{Declarations}

\section{Author contributions:}

JC, CJ, YZ, FW and DZ had full access to all the data in the study and take responsibility for the integrity of the data and the accuracy of the data. JC, FW, YZ, ZZ and QL performed the data analysis. JC, BL, YZ, and PR drafted the manuscript. JC, BL, YZ, GP and PR contributed to the study design, interpretation of the results. The authors read and approved the final manuscript.

\section{Funding}

This work was supported by Local Innovative and Research Teams Project of Guangdong Pearl River Talents Program (2017BT01S155). The sponsor had no role in the design of the study, the collection and analysis of the data, or the preparation of the manuscript.

\section{Availability of data and materials}

The datasets used and analyzed during the current study are available from the corresponding author on reasonable request. 
All participants signed a written informed consent form, and the Ethics Committee of First Affiliated Hospital, Guangzhou Medical University approved the study protocol(2013-34).

\section{Consent for publication}

Not applicable.

\section{Competing interests}

The authors declare that they have no competing interests.

\section{Acknowledgments}

We thank for Applied Chest Imaging Laboratory and Brigham and Women's Hospital for their great contribution to 3D slicer module.We thank Ryan Chastain-Gross, Ph.D., from Liwen Bianji, Edanz Group China (www.liwenbianji.cn/ac), for editing the English text of a draft of this manuscript.

\section{References}

1. Halpin D, Criner GJ, Papi A, et al. Global Initiative for the Diagnosis, Management, and Prevention of Chronic Obstructive Lung Disease. The 2020 GOLD Science Committee Report on COVID-19 and Chronic Obstructive Pulmonary Disease. Am J Respir Crit Care Med. 2021;203:24-36.

2. Pathak U, Gupta NC, Suri JC. Risk of COPD due to indoor air pollution from biomass cooking fuel: a systematic review and meta-analysis. Int J Environ Health Res. 2020;30:75-88.

3. Collaborators GOCR. Global and regional burden of chronic respiratory disease in 2016 arising from non-infectious airborne occupational exposures: a systematic analysis for the Global Burden of Disease Study 2016. Occup Environ Med. 2020;77:142-50.

4. Deng Y, Gao Q, Yang D, et al. Association between biomass fuel use and risk of hypertension among Chinese older people: A cohort study. Environ Int. 2020;138:105620.

5. Imran M, Ozcatalbas O. Determinants of household cooking fuels and their impact on women's health in rural Pakistan. Environ Sci Pollut Res Int. 2020;27:23849-61.

6. Zhou Z, Dionisio KL, Arku RE, et al. Household and community poverty, biomass use, and air pollution in Accra, Ghana. Proc Natl Acad Sci U S A. 2011;108:11028-33.

7. Siddharthan T, Grigsby MR, Goodman D, et al. Association between Household Air Pollution Exposure and Chronic Obstructive Pulmonary Disease Outcomes in 13 Low- and Middle-Income Country Settings. Am J Respir Crit Care Med. 2018;197:611-20.

8. Grajeda LM, Thompson LM, Arriaga W, et al. Effectiveness of Gas and Chimney Biomass Stoves for Reducing Household Air Pollution Pregnancy Exposure in Guatemala: Sociodemographic Effect Modifiers. Int J Environ Res Public Health 2020; 17.

9. Camp PG, Ramirez-Venegas A, Sansores RH, et al. COPD phenotypes in biomass smoke- versus tobacco smoke-exposed Mexican women. Eur Respir J. 2014;43:725-34. 
10. Zhao D, Zhou Y, Jiang C, et al. Small airway disease: A different phenotype of early stage COPD associated with biomass smoke exposure. Respirology. 2018;23:198-205.

11. Tan WC, Sin DD, Bourbeau J, et al. Characteristics of COPD in never-smokers and ever-smokers in the general population: results from the CanCOLD study. Thorax. 2015;70:822-9.

12. Camp PG, Ramirez-Venegas A, Sansores RH, et al. COPD phenotypes in biomass smoke- versus tobacco smoke-exposed Mexican women. Eur Respir J. 2014;43:725-34.

13. Xu J, Bricker J, Fu X, et al. Design and Development of Smoking Cessation Apps Based on Smokers' and Providers' Perspectives in China: Survey Study. Jmir Mhealth Uhealth. 2019;7:e12200.

14. Luo T, Li MS, Williams $D$, et al. Using social media for smoking cessation interventions: a systematic review. Perspect Public Health. 2021;141:50-63.

15. Naslund JA, Kim SJ, Aschbrenner KA, et al. Systematic review of social media interventions for smoking cessation. Addict Behav. 2017;73:81-93.

16. Lewis JJ, Pattanayak SK. Who adopts improved fuels and cookstoves? A systematic review. Environ Health Perspect. 2012;120:637-45.

17. Africa AOSO, Sciences BAO, Leopoldina GNAO, et al. Air Pollution and Health - A Science-Policy Initiative. Ann Glob Health. 2019;85:140.

18. Park J, Hobbs BD, Crapo JD, et al. Subtyping COPD by Using Visual and Quantitative CT Imaging Features. Chest. 2020;157:47-60.

19. Takayanagi S, Kawata N, Tada Y, et al. Longitudinal changes in structural abnormalities using MDCT in COPD: do the CT measurements of airway wall thickness and small pulmonary vessels change in parallel with emphysematous progression? Int J Chron Obstruct Pulmon Dis. 2017;12:551-60.

20. Tanimura K, Sato S, Fuseya Y, et al. Quantitative Assessment of Erector Spinae Muscles in Patients with Chronic Obstructive Pulmonary Disease. Novel Chest Computed Tomography-derived Index for Prognosis. Ann Am Thorac Soc. 2016;13:334-41.

21. Diaz AA, Martinez $\mathrm{CH}$, Harmouche R, et al. Pectoralis muscle area and mortality in smokers without airflow obstruction. Respir Res. 2018;19:62.

22. Charbonnier JP, Pompe E, Moore $\mathrm{C}$, et al. Airway wall thickening on CT: Relation to smoking status and severity of COPD. Respir Med. 2019;146:36-41.

23. Liu S, Zhou Y, Liu S, et al. Association between exposure to ambient particulate matter and chronic obstructive pulmonary disease: results from a cross-sectional study in China. Thorax. 2017;72:78895.

24. Zhou Y, Zou Y, Li X, et al. Lung function and incidence of chronic obstructive pulmonary disease after improved cooking fuels and kitchen ventilation: a 9-year prospective cohort study. Plos Med. 2014;11:e1001621.

25. Boueiz A, Chang Y, Cho MH, et al. Lobar Emphysema Distribution Is Associated With 5-Year Radiological Disease Progression. Chest. 2018;153:65-76. 
26. Matsuoka S, Washko GR, Yamashiro T, et al. Pulmonary hypertension and computed tomography measurement of small pulmonary vessels in severe emphysema. Am J Respir Crit Care Med. 2010;181:218-25.

27. Suzuki Y, Yoshimura K, Enomoto Y, et al. Distinct profile and prognostic impact of body composition changes in idiopathic pulmonary fibrosis and idiopathic pleuroparenchymal fibroelastosis. Sci Rep. 2018;8:14074.

28. Lin H, Xiao D, Liu Z, et al. National survey of smoking cessation provision in China. Tob Induc Dis. 2019;17:25.

29. Leone FT, Zhang Y, Evers-Casey S, et al. Initiating Pharmacologic Treatment in Tobacco-Dependent Adults. An Official American Thoracic Society Clinical Practice Guideline. Am J Respir Crit Care Med. 2020;202:e5-31.

30. Wang Z, Chen X, Liu K, et al. Small pulmonary vascular alteration and acute exacerbations of COPD: quantitative computed tomography analysis. Int J Chron Obstruct Pulmon Dis. 2016;11:1965-71.

31. Cho YH, Lee SM, Seo JB, et al. Quantitative assessment of pulmonary vascular alterations in chronic obstructive lung disease: Associations with pulmonary function test and survival in the KOLD cohort. Eur J Radiol. 2018;108:276-82.

32. Juntarawijit C, Juntarawijit Y. Cooking with biomass fuel and cardiovascular disease: a crosssectional study among rural villagers in Phitsanulok. Thailand F1000Res. 2020;9:307.

33. Salvi SS, Brashier BB, Londhe J, et al. Phenotypic comparison between smoking and non-smoking chronic obstructive pulmonary disease. Respir Res. 2020;21:50.

34. Chan KH, Kurmi OP, Bennett DA, et al. Solid Fuel Use and Risks of Respiratory Diseases. A Cohort Study of 280,000 Chinese Never-Smokers. Am J Respir Crit Care Med. 2019;199:352-61.

35. Lim JU, Kim EK, Lim SY, et al. Mixed Phenotype of Emphysema and Airway Wall Thickening Is Associated with Frequent Exacerbation in Chronic Obstructive Pulmonary Disease Patients. Int J Chron Obstruct Pulmon Dis. 2019;14:3035-42.

36. McDonald ML, Diaz AA, Ross JC, et al. Quantitative computed tomography measures of pectoralis muscle area and disease severity in chronic obstructive pulmonary disease. A cross-sectional study. Ann Am Thorac Soc. 2014;11:326-34.

\section{Figures}




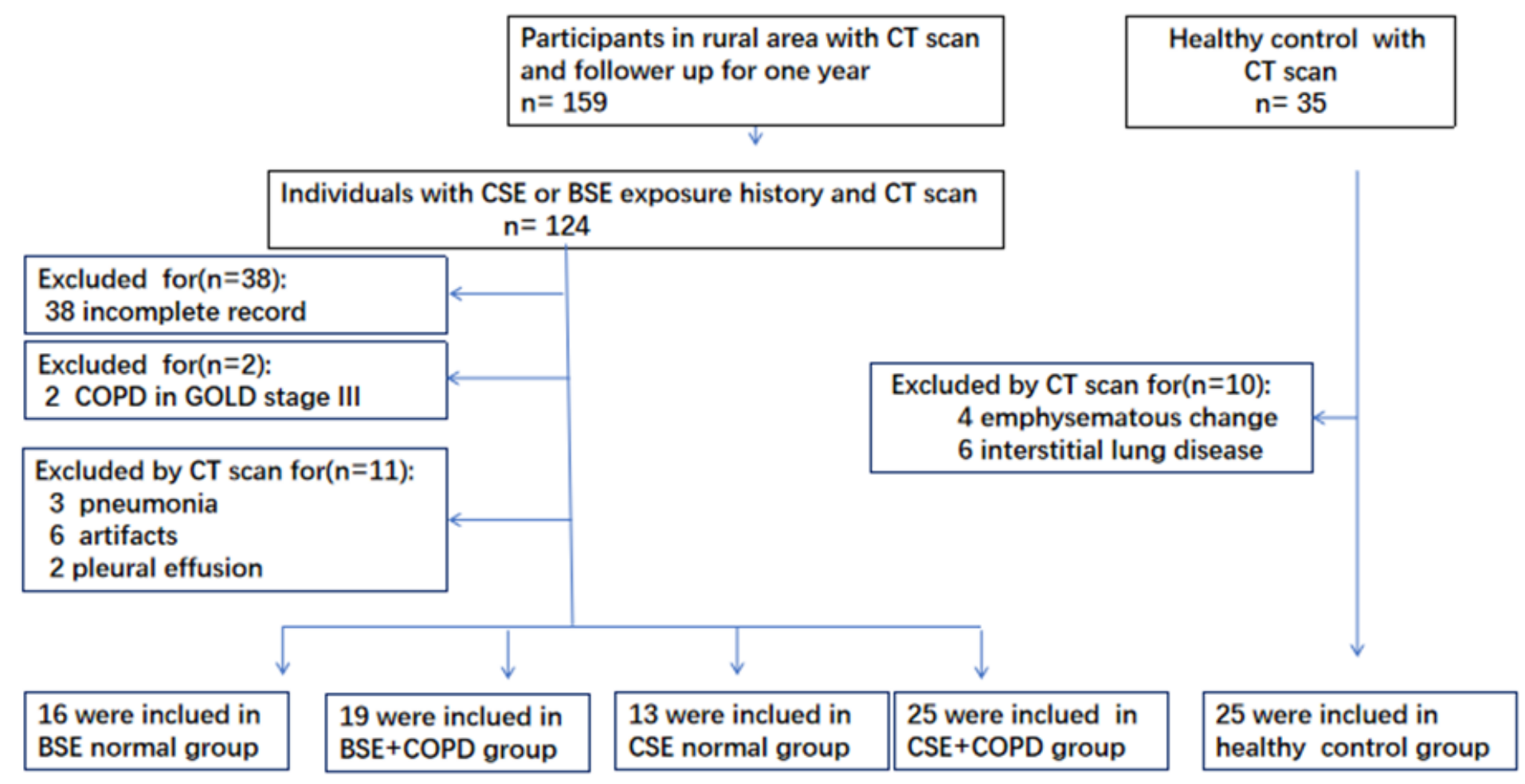

\section{Figure 1}

Flow chart of the study participants 
a

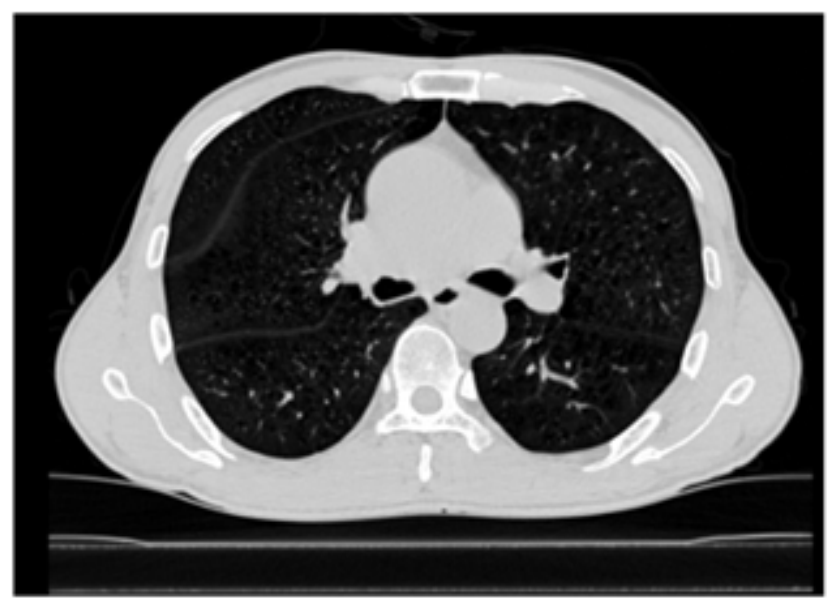

C

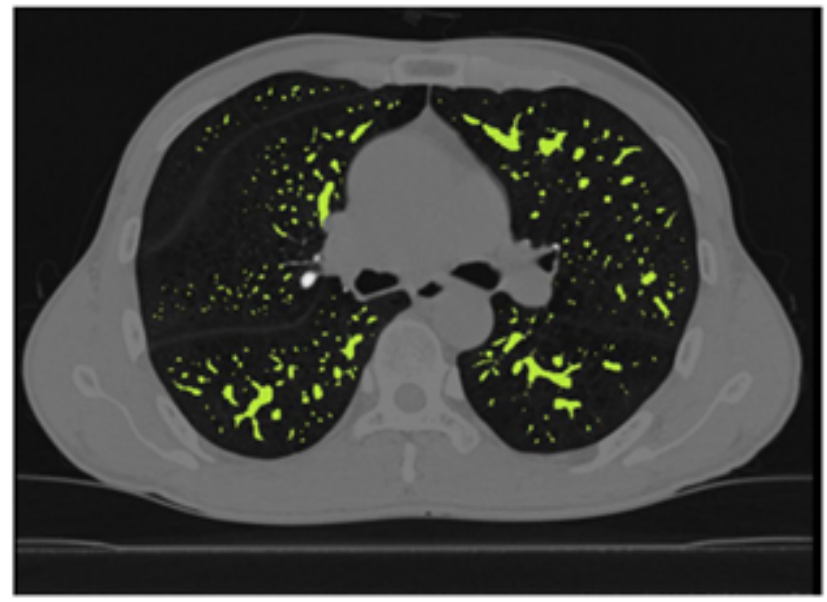

b

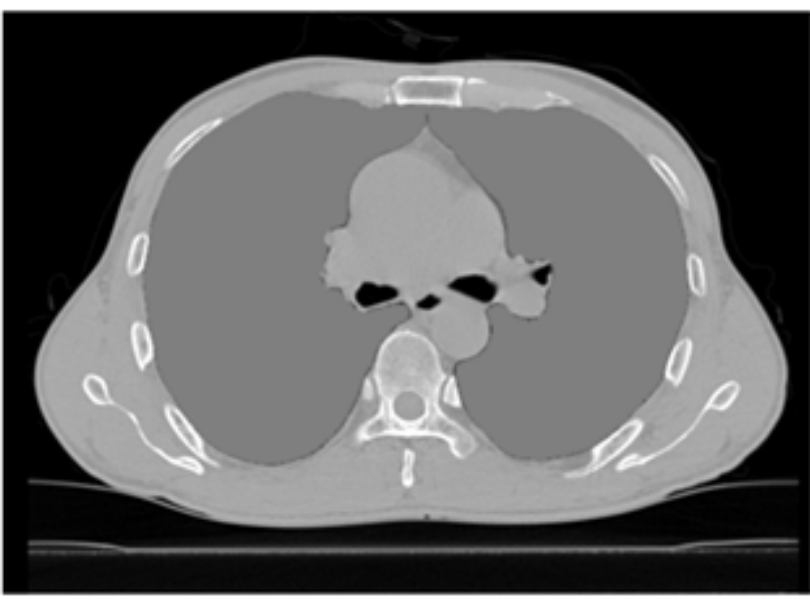

d

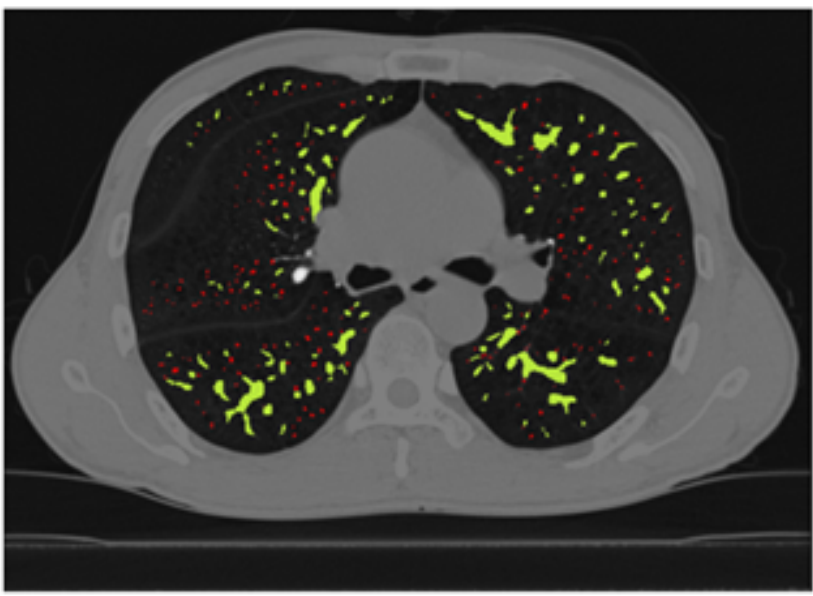

Figure 2

Sample computed tomography (CT) scans used to determine pulmonary small vessels a CT image in middle slice of lung. b Segmented lungs shaded in grey. c Pulmonary vessels shaded in green. $d$ pulmonary small vessels shaded in red.

A

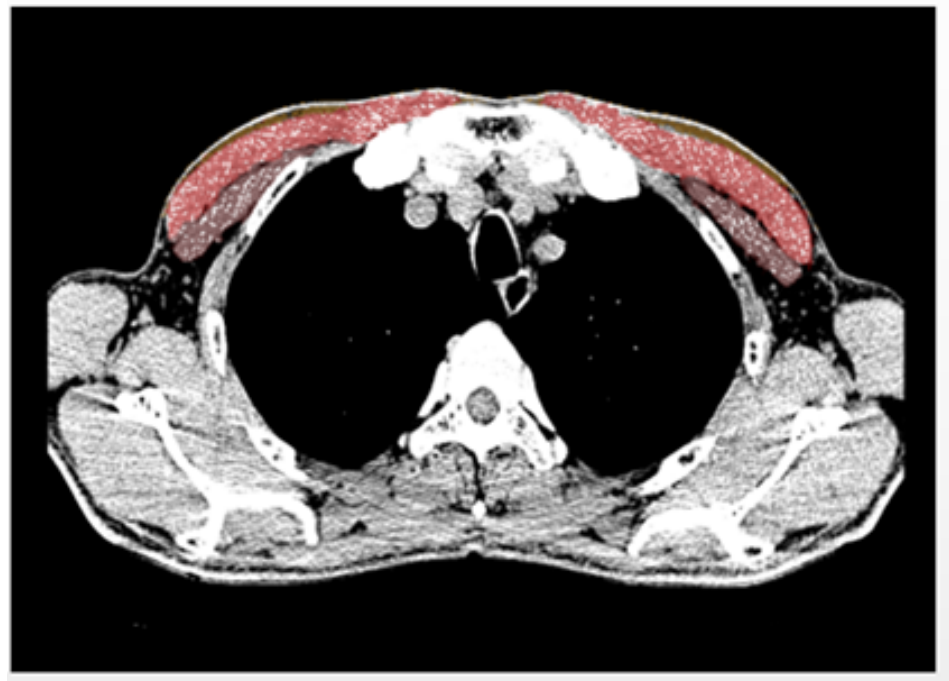

B

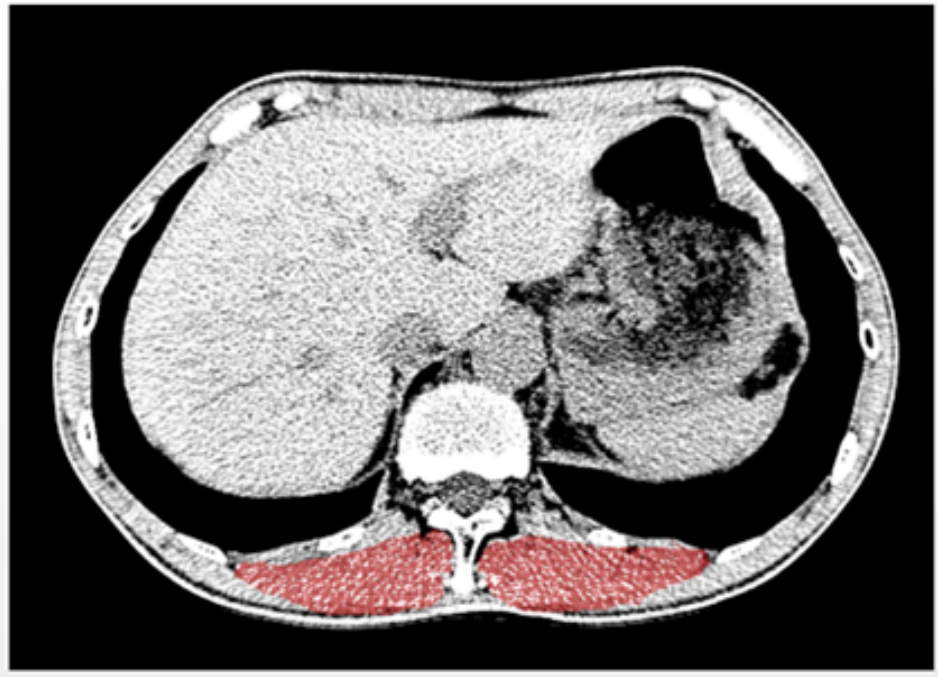




\section{Figure 3}

Sample computed tomography (CT) scans used to determine pectoralis muscle, erector spinae muscle a Pectoralis major muscle shaded in red, pectoralis minor muscle shaded in brown. b Erector spinae muscle shaded in red.

\section{Supplementary Files}

This is a list of supplementary files associated with this preprint. Click to download.

- SupplementaryTable.doc 